INTERNATIONAL DESIGN CONFERENCE - DESIGN 2018

https://doi.org/10.21278/idc.2018.0141

\title{
THE FUNCTION OF CO-CREATION IN DYNAMIC MECHANISM OF INTERSUBJECTIVITY FORMATION AMONG INDIVIDUALS
}

\author{
A. Matsumae and Y. Nagai
}

\begin{abstract}
This study aims to elucidate the dynamic mechanism of formation process of intersubjectivity among individuals in various contexts: how a design process affects formation of intersubjectivity among individuals involved and also their co-creativity, motivation toward realization of the co-designed concept. The authors have defined key terms and proposed methodologies to evaluate fundamental factors of co-creation dynamics, intersubjectivity, context, and co-creativity. Then to examine them, an experimental study was conducted concerning the type of collaborative design process.
\end{abstract}

Keywords: co-design, collaborative design, design creativity, intersubjectivity, dynamic model

\section{Introduction}

\subsection{Background}

The importance of collaboration among diverse individuals and/or organizations for innovation is often emphasized (Sawyer, 2011), but collaboration does not happen just by having individuals together at a certain place. It is also lamentable to see collaborative projects wound up when the given framework is taken away, for instance, facing a loss of resources, becoming obsolete in dynamic circumstances, or the termination of a period of subsidy.

Regardless the fields applied, there have been substantial studies on the collaboration process from the points of view of knowledge sharing, knowledge integration and shared understanding in established teams (Maier et al., 2009; Gendron et al., 2012; Kleinsmann et al., 2012; Yang et al., 2012; Cash et al., 2017). However, these are not enough to understand co-creative collaboration since it requires more consideration of internal human factors than co-operative collaboration.

Therefore, it is important to form autonomic intersubjectivity for sustainable innovation, and to develop a methodology for its design and management.

\subsection{Related studies}

\subsubsection{Definition of co-creativity}

The definition of co-creativity in this study can be understood by referring to the recently published literature on creativity cited below. The standard definition of creativity has consistently required two attributes of originality and effectiveness though they are paraphrased into different expressions by different researchers (Runco and Jaeger, 2012). Based on the understanding of creativity generated within an individual, the research on creativity generated among individuals has been developed by 
researchers (Sawyer, 2011; Gilson et al., 2015). For instance, research on group creativity can be seen in specific fields such as music and drama (St. John, 2007; Chen, 2017). As more generalized research, organizational creativity was defined as the creation of a valuable, useful new product, service, idea, procedure, or process by individuals working together in a complex social system (Woodman et al., 1993).

However, this literature, which assume a stable organization as a subject for co-creation, overlook a fundamental difference between co-creativity and creativity; the unstable basis of co-creativity. Cocreativity is generated among individuals in contrast to creativity generated within an individual, and it means that co-creativity is founded on a fragile basis. This point of view is especially important in the context of the fuzzy front-end phase (Reid and De Brentani, 2004), which the authors focus on in this study. Considering that both the essential factors of creativity, effectiveness and novelty, can be improved through the continuous creative process and that it is motivation, which initiates and sustains the creative process (Amabile, 1983), the necessary condition for co-creation can be said to maintain shared motivation among individuals to develop their project for the better.

Thus, the definition of co-creativity has evolved in this study to mean a shared motivation among individuals to develop and realize their concept.

\subsubsection{Definition of co-creation}

The term co-creation is widely used as an important keyword in knowledge creation. However, it has not been clearly defined and the difference among collaboration, co-creation and co-operation is ambiguous. In this study, referring to the SECI spiral (Nonaka and Takeuchi, 1995), the authors define co-creation as the collaboration to create something together, sharing the phase of socialization among individuals. Intersubjectivity among stakeholders is formed as the basis of co-creation by sharing socialization, which is the process of converting tacit knowledge to new tacit knowledge through shared experiences and social interaction (Nonaka and Takeuchi, 1995). Sharing a clear common goal among individuals is not required at the beginning of the co-creation process, but appears throughout the process of dynamically developed co-creation.

On the other hand, we define co-operation, distinctive from co-creation, as the collaboration to achieve a given common goal without sharing the phase of socialization among individuals. A given common goal must be central among individuals from the beginning for co-operation (Figure 1).

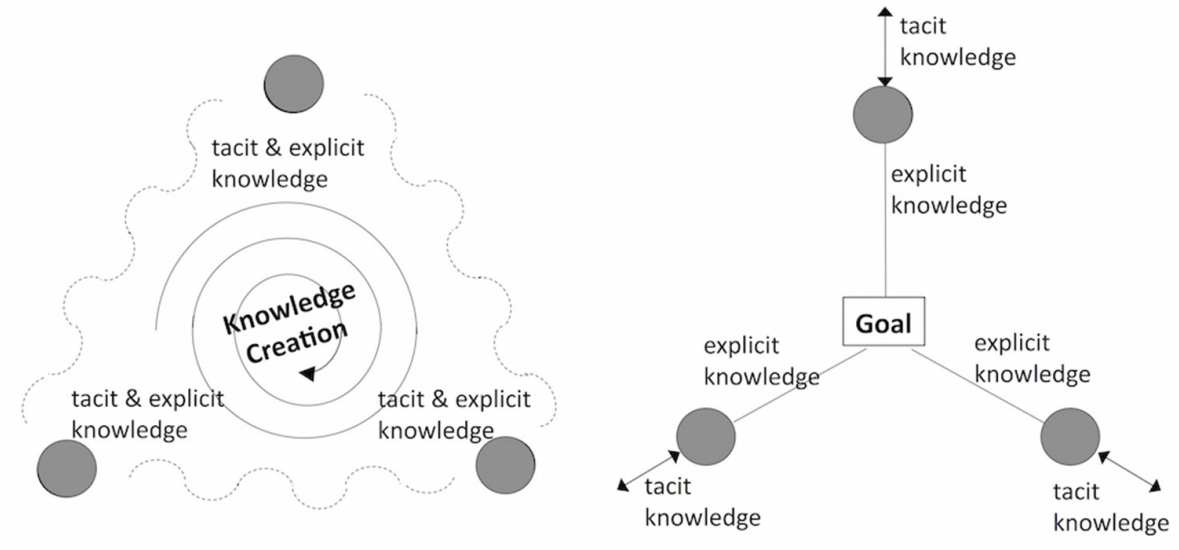

Co-creation Co-operation

Figure 1. Comparison between co-creation and co-operation

\subsubsection{Intersubjectivity}

From accumulated preliminary case studies, authors regard intersubjectivity as an essential factor to generate and to sustain collaborative project (Matsumae, 2014; Matsumae and Burrow, 2016). The concept on intersubjectivity has its origin in Husserl's phenomenology and has been developed into 
several different directions focused on its different aspects. The domain of intersubjectivity lies beneath the empathy of the Husserl's Fifth Cartesian Meditation (Zahavi, 2001; Donohoe, 2016), and the basic idea of intersubjectivity is that subjects do not constitute a world alone, but jointly, together with other subjects; this world lies beneath the empathy of the Fifth Cartesian Meditation (Bower, 2014).

The importance of intersubjectivity from the aspect of knowledge co-creation is pointed to as a source of motive power for execution (Nonaka and Konno, 2012), however it is difficult to find experimental research to support their indication because of its complexity of the phenomena or difficulty in capturing and measuring human's internal status. Tomasello observed infants' behaviour and found that intersubjectivity is not only responsive to other subjects' goals, but also the means employed in achieving those goals (Tomasello, 2008). There is a recent experimental study, which captures shared understanding among team members during collaboration (Cash et al., 2017), but shared understanding is not based on intersubjectivity among members, but on each member's independent subjectivity.

In this paper, the authors propose a methodology to evaluate intersubjectivity being formed among individuals and indicate knowledge obtained from an experimental study focused on relationships between intersubjectivity and the collaborative process.

\subsection{Aim of this study}

The purpose of this study is to obtain knowledge concerning how a design process affects (1) formation of intersubjectivity among individuals involved, and also (2) their motivation toward realization of the co-designed concept.

The authors extracted following fundamental viewpoints in this experiment; 1) types of design process, 2) degrees of formation of intersubjectivity, and 3) design contexts.

\section{Methodology}

The fundamental factors that have been considered in this study are extracted mainly through the observations from accumulated experimental and practical case studies, followed by related past studies as explained under the Section 1.2. With the purpose of examining the behaviour of each identified factor in a simple realistic situation, the experiment, "designing a pizza for Little Red Riding Hood to bring to her sick grandmother" has been carried out. The behaviour of each factor has been measured as explained under the Section 2.1 and the co-creation dynamics model has been proposed based on the results obtained as mentioned in the Section 5.1.

\subsection{Viewpoints of evaluation}

Since both design and creation are activities arising from humans' internal status, it is essential to capture the dynamic nature of this internal status to understand the mechanism of co-creation. Whether measured directly or indirectly, internal status cannot be captured perfectly with a single measurement. Results can differ depending on who is evaluating, when measurements are taken concurrently or ex-post (Nagai et al., 2010). However, it is also true that each result reveals a portion of the major tendency of internal status. For example, internal status can be captured partly by observing a subject's external appearance and also by the self-description based on their own awareness. Therefore, the authors took a multipronged approach to capture the subject's internal nature with higher precision, in the same way a court captures a case along with its established law methodology, reasoning from duplicated indirect facts with evidence from different points of view. At the same time, the authors regard it as important to choose the appropriate evaluation scale considering the complexity and the fluctuation of human nature and cognitive ability. To capture the dynamics of internal human nature, intersubjectivity (Section 2.2), contexts (Section 2.3) and co-creativity (Section 2.4) were chosen as essential factors in this study and evaluated.

\subsection{Evaluation of intersubjectivity}

Intersubjectivity is evaluated based on self-description of internal nature by participants and on observation of external appearance in reasonably appropriate precision by experts. 


\subsubsection{Self-description by participants}

Each of the participants was requested to record one's own cognition of one's nature dynamics. To capture the formation of intersubjectivity, the qualitative coincidence of fluctuations, in other words, emotional wave patterns, is analysed by comparing the customer journey maps of all participating individuals. In contrast, it is not important to meet quantitative coincidence, since each individual has a different degree of intensity in his/her expression.

The challenge evaluating internal human nature as numerical information is significant, however too detailed numerical calculations are counter-productive and fail to reflect the true status. An appropriate range of precision according to the nature of research object, intersubjectivity among different individuals is required. The authors carefully duplicated evaluation methods to capture each participant's emotional fluctuation to see the degree of qualitative coincidence of fluctuation as a state of intersubjectivity being formed among them, pursuing higher precision while avoiding meaninglessly detailed numerical calculations.

\section{Pattern Comparison}

One evaluation method is a simple categorization of wave patterns, which is captured as a combination of three modes (increasing, unchanging, or decreasing). For instance, when an observation term is divided into two periods, wave patterns can be categorized into nine patterns (Figure 2): increasing-increasing, increasing-unchanging, increasing-decreasing, unchanging-increasing, unchanging-unchanging, unchanging-decreasing, decreasing-increasing, decreasing-unchanging and decreasing-decreasing.
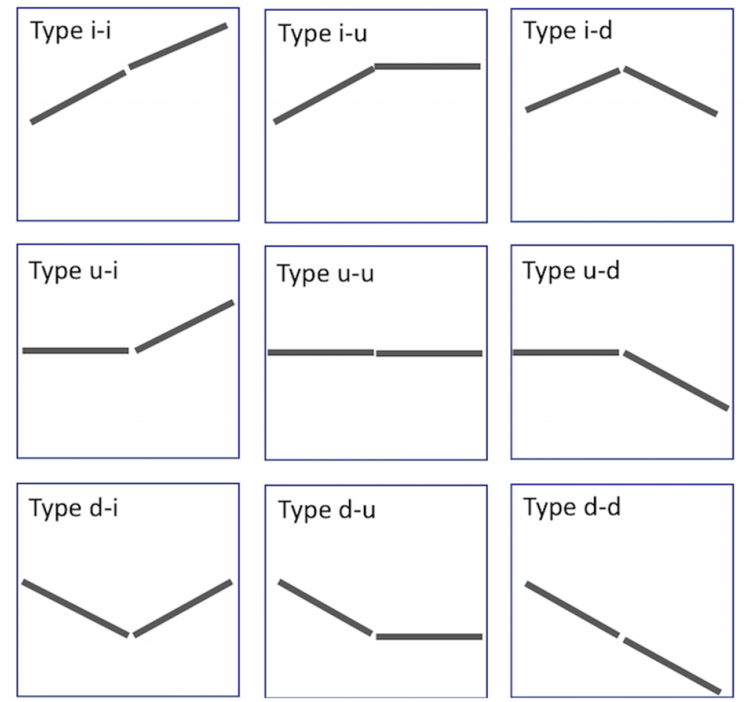

Figure 2. Examples of simplified categorization of wave pattern

\section{Quantitative Comparison}

The other evaluation method is a quantitative comparison of each participant's emotional waves. Numerically self-described emotional waves are normalized for pattern recognition according to each participant's maximum amplitude throughout the experiments, and also appropriately simplified considering the precision of cognition ability of participants.

For instance, let $f_{P}$ be a continuous function such that $f_{P}(t)$ represents the person $P$ 's original emotional value at time $t$. The function $\widehat{f}_{P}$ is defined as the normalized function of $f_{P}$, considering the individual difference of emotional amplitude. The function $\widehat{f}_{P}$ is formally expressed as,

$$
\widehat{f}_{P}(t) \stackrel{\text { def }}{=}-1+2 \frac{f_{P}(t)-m_{P}}{M_{P}-m_{P}}
$$

where $M_{P}=\max _{t} f_{P}(t)$ and $m_{P}=\min _{t} f_{P}(t)$. Obviously, it is not difficult to check the following three conditions hold for $\widehat{P_{P}}$ : 


$$
\begin{aligned}
& \forall t\left[-1 \leq \widehat{f}_{P}(t) \leq 1\right], \\
& \forall t\left[\left(f_{P}(t)=M_{P}\right) \Rightarrow\left(\widehat{f}_{P}(t)=1\right)\right], \text { and } \\
& \forall t\left[\left(f_{P}(t)=m_{P}\right) \Rightarrow\left(\widehat{f}_{P}(t)=-1\right)\right] .
\end{aligned}
$$

On the other hand, $f_{P}(t)$ can be approximated as a discrete function $\widetilde{f}_{P}(t)$ defined over a discrete series of time points $t_{1}, t_{2}, \ldots, t_{N}$, where each $t_{i}$ is defined with an appropriate time interval so that every inflection points of $f_{P}$ is included in the domain of $\widetilde{f}_{P}$.

Then the degree of intersubjectivity formation among individuals $P_{1}, \ldots, P_{n} \operatorname{Isub}\left(P_{1}, \ldots, P_{n}\right)$, can be defined as follows:

$$
\operatorname{Isub}\left(P_{1}, \ldots, P_{n}\right) \stackrel{\text { def }}{=} \frac{1}{n N} \sum_{t=t_{1}}^{t_{N}}\left(\left|f_{P_{1}(t)}-A v e_{(t)}\right|^{2} \ldots+\left|f_{P_{n}(t)}-A v e_{(t)}\right|^{2}\right)
$$

where $A v e_{(t)}=\frac{1}{n} \sum_{i}^{n} f_{P i(t)}$.

\subsubsection{Observation by experts}

Three experts observed each team from external appearance throughout the experiment. All of the experts have more than fifteen-year experience evaluating team projects. They were asked to observe and record participants' behaviour focusing on how inter-subjectivity was formed in each team along with their own professional point of view.

\subsection{Evaluation of context}

In order to track a dynamic change of design context (Shalley et al., 2004) for each individual and team, the authors introduced the concept of context vector. A context is expressed in vector form in this study based on self-descriptions of participants. Each word shown in all descriptions is categorized and each category is given a different coordinate axis.

For instance, when all of words found in all self-descriptions can be categorized into $n$ categories, the $\overrightarrow{C t x}(P)$, context vector of person $P$, can be expressed,

$$
\overrightarrow{C t x}(P) \stackrel{\text { def }}{=}\left(F_{P}(1), \ldots, F_{P}(n)\right)
$$

where $F_{P}(i)$ is a context force effected on the person $P$ in category $i$.

Then the distance between contexts $\overrightarrow{C t x}(P)$ and $\overrightarrow{C t x}(Q)$ can be defined as follows:

$$
\operatorname{DIST}(\overrightarrow{\operatorname{Ctx}}(P), \overrightarrow{\operatorname{Ctx}}(Q)) \stackrel{\text { def }}{=} \sqrt{\left|p_{1}-q_{1}\right|^{2} \ldots+\left|p_{n}-q_{n}\right|^{2}}
$$

where $\overrightarrow{C t x}(P)=\left(p_{1}, \ldots, p_{n}\right)$ and $\overrightarrow{C t x}(Q)=\left(q_{1}, \ldots, q_{n}\right)$.

\subsection{Evaluation of co-creativity}

As described in Section 1.1.2, co-creativity in this study is defined as a shared motivation among individuals to realize and develop their concepts. As an absolute evaluation of co-creativity, each participant is asked to express one's degree of motivation toward realization of each concept as a percentage. As a relative evaluation, each participant is asked to order these concepts, to compare more clearly the effect of design process on co-creativity generation among individuals.

\section{Experiment}

\subsection{Participants}

Thirty professionals from various occupations participated in this experiment. Participants were divided into six teams, from A to F, and each team had five members, numbered, for example, as A01, through A05 in team A. Team composition was carefully determined and the authors confirmed in advance that no members of the same team had any previous knowledge of other team members, to examine the formation process of inter-subjectivity in the experiment without the influence of existing relationships. 
Each team was also composed to maximize diversity in terms of gender, age, occupational category, and rank in the organizations they belong to.

\subsection{Procedure}

The procedure of the experiment has three processes, introduction (W00), co-operative work (W01) and co-creative work (W02) (Figure 3). After each process, participants were required to record their internal nature in five minutes. Considering cognitive precision, participants were instructed to record their internal nature on a 5-point Likert scale $(-2,-1,0,+1,+2)$ at the beginning, middle, and end of each period in this experiment and record short explanations as to their internal nature. Participants were also asked to record their motivation toward realization for each concept as described in Section 2.4 at the end of the series of works. Experts were asked to observe and record participants' behaviour and each team's degree of formation of intersubjectivity in real time along a continuous time axis throughout the experiment as described in Section 2.2.2. This experiment was also recorded as video for ex-post evaluation.
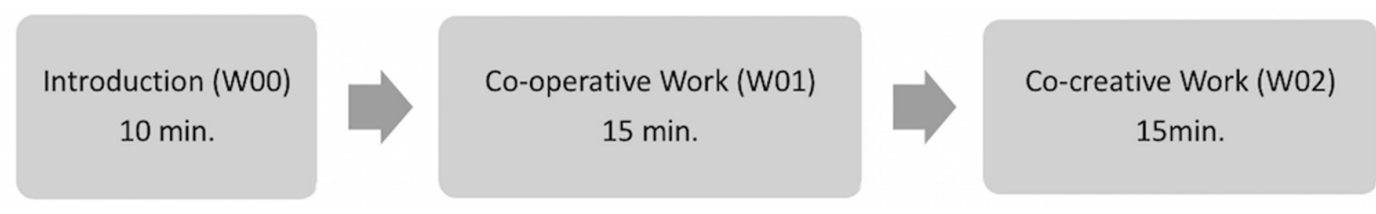

Figure 3. Procedure of experiment

\subsubsection{Introduction (W00)}

Participants were led to their designated table by team and given 10 minutes to introduce themselves to each other. Then they were given information that they would be assigned a set of collaborative design work as a team: designing a pizza for Little Red Riding Hood to bring to her sick grandmother. Even though every participant knew the story of Little Red Riding Hood, they were reminded of the outline of the story to ensure uniform knowledge of the story before the work. Identical sets of materials for the experiment such as 10-colour-clay and pizza design template sheets had been prepared for each table in advance. At the end of the exercise, the evaluation procedure was explained and each participant was asked to record the transition of his/her internal nature as described in the beginning of Section 3.2. No collaborative work was requested of participants during W00. W00 was designed not only to prepare for a set of collaborative design works but also to capture the basic status of participants.

\subsubsection{Co-operative design work (W01)}

As a co-operative design work, each of participants was required to design a pizza in five minutes using a simplified design template. Then each team chose one pizza design out of the five by rolling dice to make into clay-pizza. Participants were requested to co-operate in each team to make a clay-pizza strictly following the chosen design in the following ten minutes.

\subsubsection{Co-creative design work (W02)}

As in W01 above, as a co-creative design work rather than cooperative design work, participants were requested to design a pizza together in five minutes in teams using the same simplified design template as the one for W01. However, in W02, the design was not chosen by the roll of dice, but co-designed by all members in the team. Then participants were requested to make a clay-pizza together by team based on their own design in the following ten minutes. They were allowed to change their original design during their clay work.

\section{Results}

\subsection{Intersubjectivity}

To evaluate the intersubjectivity being formed among individuals, degrees of coincidence of each participant's emotional waves are examined in two ways: pattern comparison (Section 4.1.1) and 
quantitative comparison (Section 4.1.2). The evaluation methodology described in Section 2.2 was applied to obtained data from the experiment described in Section 3.2.

\subsubsection{Pattern comparison}

The results of each team's simplified pattern comparison are shown in Figure 4 (A to E) below. There are two sets of lines for each team, an emotional wave for co-operative work (W01) placed on the left and one for co-creative work (W02) on the right. Participant D03 withdrew due to illness, and her data have been excluded from the calculation.

From simplified pattern comparison, the major tendency concerning intersubjectivity can be captured visually; (1) wave patterns have less variety in W02 than W01, (2) these results match well with the experts' evaluation on intersubjectivity; for example, "Team C lacked a sense of unity during W01 but rapidly formed it and then worked dividedly in W02.", "Team E enjoyed both works individually, didn't challenge co-creation in W02 and failed to form intersubjectivity throughout the experiment," or "Team A formed intersubjectivity the earliest and kept it until they finished W02."

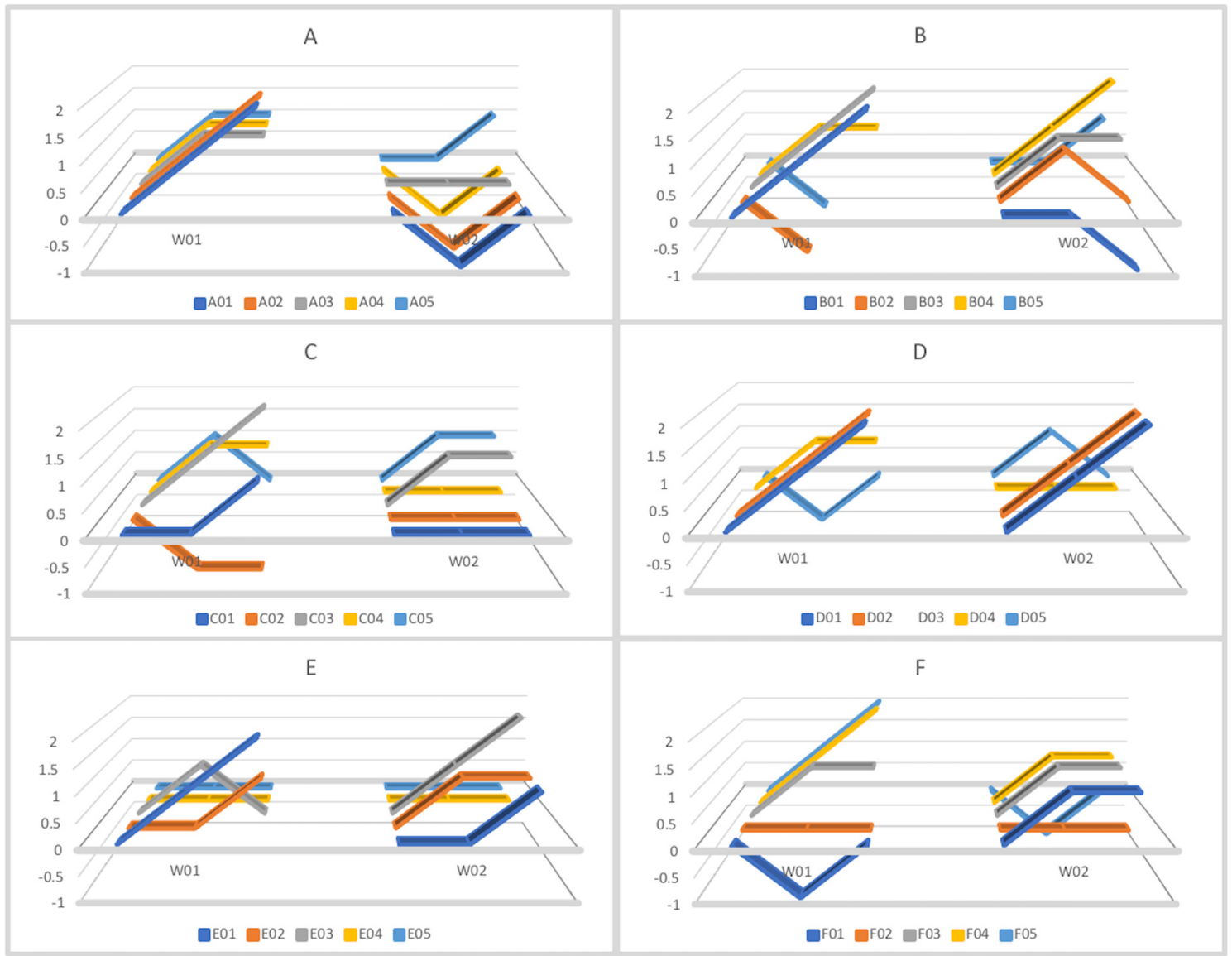

Figure 4. Pattern comparison between W01 (left) and W02 (right)

\subsubsection{Quantitative comparison}

To capture the quantitative variance of intersubjectivity, the authors applied the methodology described in Section 2.2 to data obtained from this experiment. According to our definition, the more intersubjectivity is developed, the less variance of intersubjectivity occurs. The results are shown in Figure 5. The calculation of the t-test showed that this result of differences between W01 and W02 for the variance of intersubjectivity is statistically significant $(t=1.95, \mathrm{df}=5, p=0.05)$.

Except team E, where co-creation was not tried during W02 as written in 4.1.1, the variance of intersubjectivity decreased from W01 to W02 and converged below 0.8 in W02. Here again results match 
well with the experts' evaluation on intersubjectivity; for example, experts observed from team B that continuous interactive communication among individuals accelerates the formation of intersubjectivity.

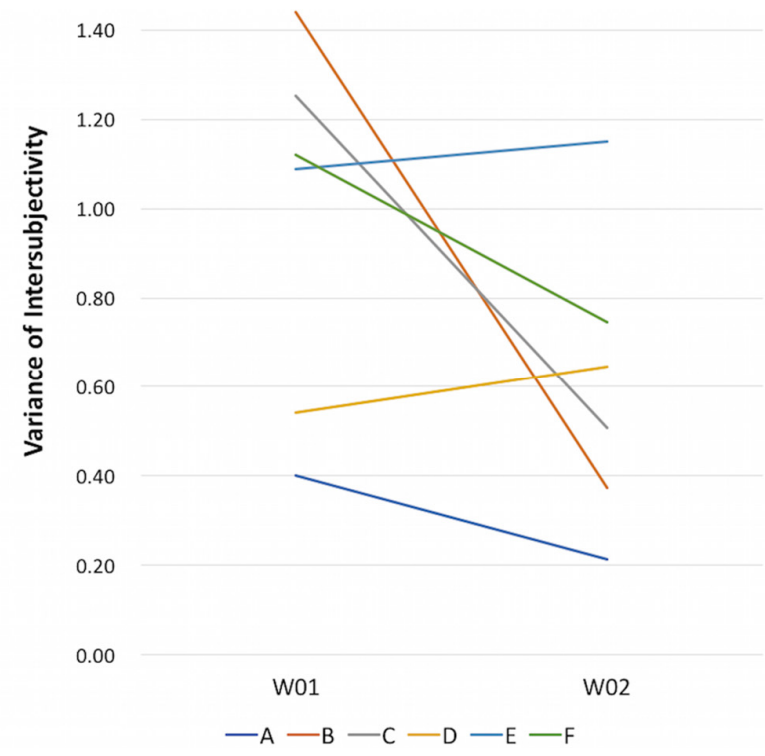

Figure 5. Quantitative comparison on variance of intersubjectivity

\subsection{Context}

All words self-description by participants as their design context could be categorized into eleven dimensions; such as "nutritious", "soft", "colourful", "grandma's favourites".

Figure 6 shows the sum of distances in each team between the reference context and each participant's context in W01 and W02. The t-test showed that this result of differences between W01 and W02 in relation to the sum of the distance of context vectors is statistically significant $(t=2.95, d f=5, p=0.032)$. It is clear that the sum of the distance of context vectors decreased from W01 to W02, except for team E where co-creation was not attempted during W02. The contexts of individuals converged through the co-creation process, on the other hand that co-creation process is led by their contexts.

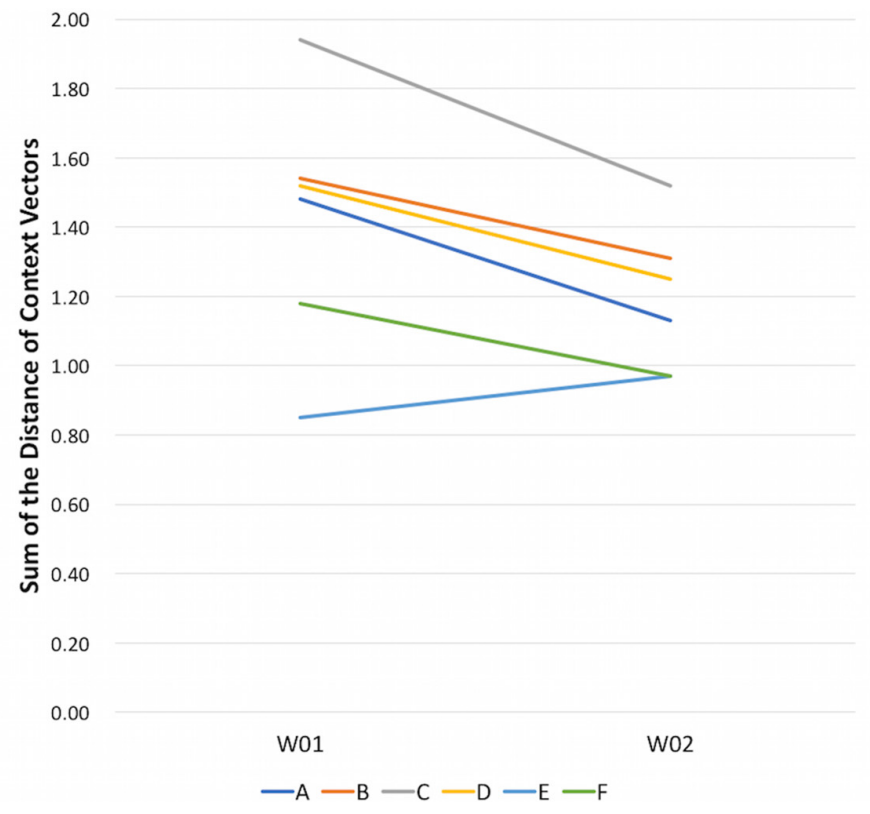

Figure 6. Sum of the distance of context vectors 


\subsection{Co-creativity}

As described in Section 1.1.2, co-creativity in this study is defined as a shared motivation among individuals to realize and develop their concepts. As expected, the relative evaluation of co-creativity described in Section 2.4 indicates a clear tendency to formation of intersubjectivity. In teams A, B, C and $\mathrm{F}$, where the variance of intersubjectivity decreased from W01 to W02, all participants answered that they want to realize the pizza concept co-created in W02 more than the one co-operated in W01. On the contrary, 2 of 5 in team D and 3 of 5 in team E, where the variance of intersubjectivity increased from W01 to W02 answered that they want to realize the pizza concept in W01 more than the one in W02.

From the absolute evaluation of co-creativity described in Section 2.4, Co-creativity Index, the ratio of members who want to realize the concept co-created in a team, is calculated for each team and for each work, W01 and W02. The relation between the Co-creativity Index and the variance ratio of intersubjectivity is indicated below in Figure 7. It shows that the more intersubjectivity converged among individuals, the stronger co-creativity was shared in a team.

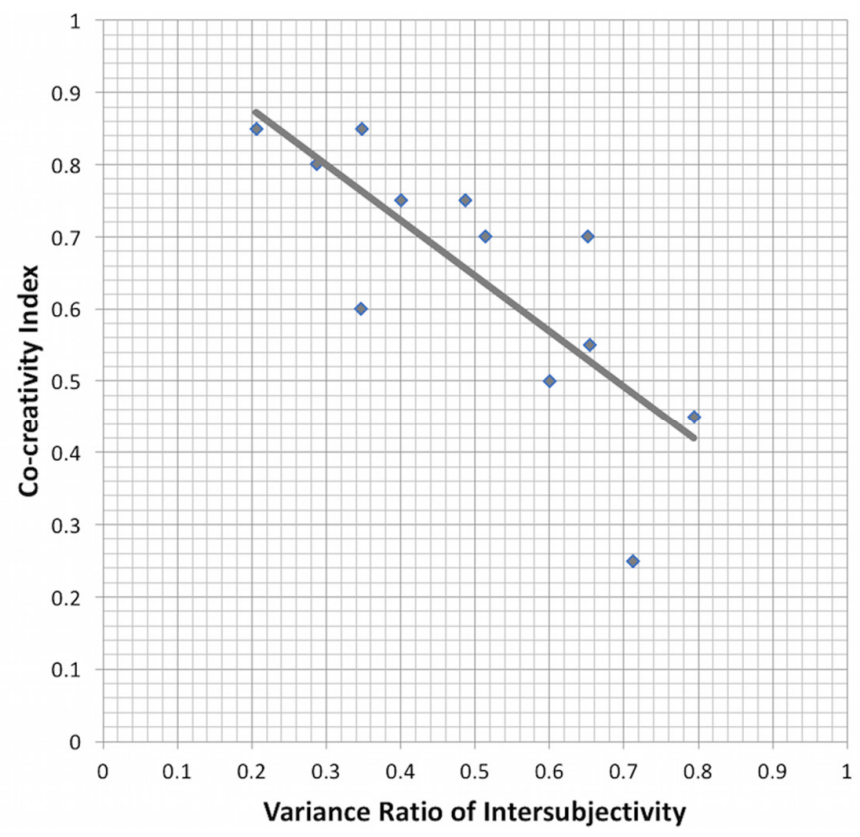

Figure 7. Relation between variance of intersubjectivity and co-creativity

\section{Discussions}

\subsection{Proposition of model for co-creation dynamics}

The authors placed the key factors into a co-creation dynamic system named inverted vortex model (IVM), which represents the results of this study (Figure 8). This model indicates that individuals with various contexts form intersubjectivity through co-creation, as moving objects, black points in Figure 8, converge at a deeper point along with the fluid flow of the vortex under appropriate conditions. Cocreation process and individuals' context interact with each other as a vortex and moving objects interact each other: the objects converge in the vortex, and moving objects are transformed, occasionally breaking the vortex.

As co-creativity is defined to mean a shared motivation among individuals to develop and realize their concept in Section 1.2.1, elements of IVM seem to parallel Amabile's elements of motivation. Amabile indicated that motivation includes two elements: the individual's base line attitude toward the task and the individual's perceptions of his or her reasons for undertaking the task. Baseline attitude is explained as the degree to which it matches his or her existing preferences and interests, and perceptions are proposed that depend largely on external social and environmental factors (Amabile, 1983). The 
boundary object in the authors' IVM has a similar role to Amabile's baseline attitude since the boundary object indicates the boundary of an individual's existing preference and interests. Likewise, context in IVM works similarly to perception since it leads to individual's creativity.

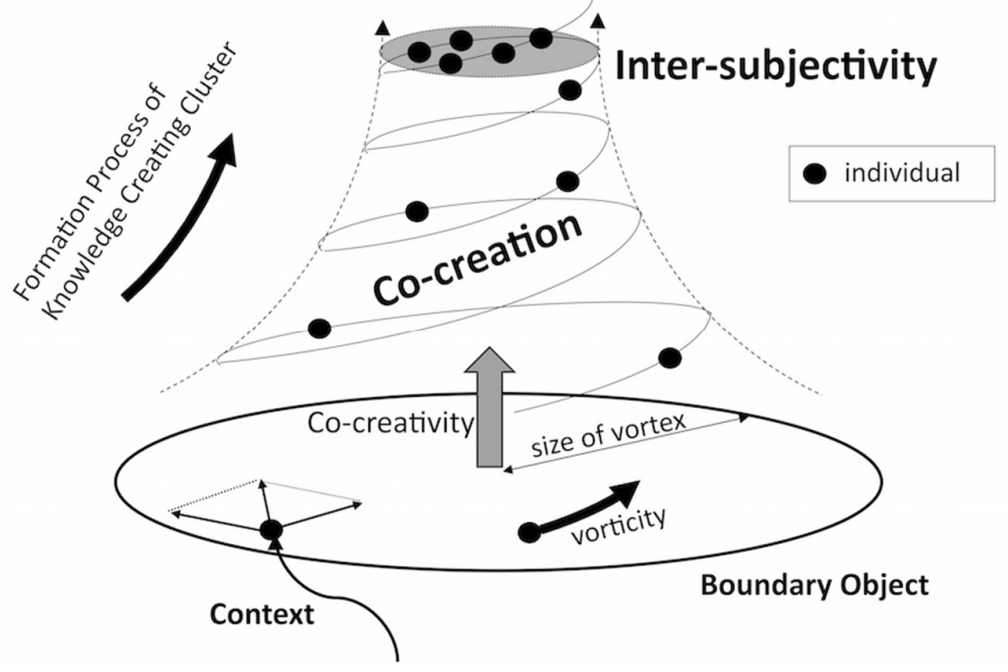

Figure 8. Inverted Vortex Model for co-creation dynamics

\section{Boundary Object}

A boundary object is originally advocated as an object that is adaptable to multiple viewpoints and robust enough to maintain identity across them (Star and Griesemer, 1989; Star, 2010). In later studies, the boundary object is scaled up as a sort of arrangement that allows different groups to work together without consensus, especially in the context of collaboration infrastructure (Star, 2010).

By setting an appropriate boundary object and co-creation process in the project, different contexts of each individual can be bundled and sharpened. Focusing its function to involve different contexts in this particular co-creation, the authors introduce two concepts to characterize a boundary object from the analogy of Rankine's combined vortex: 1) the size of a boundary object to describe its reach and 2) vorticity of the boundary object to describe how it works in each individual.

\section{Context}

Creation exists in each creator's contexts. Some contexts can be described in same dimension, but others can be described in different dimensions. Each individual has its own original context as a creator. A context can be modified through interaction with other contexts, and contexts can generate a new context. The authors introduce the concept of context force vector to describe the direction and the magnitude of each creators' context. A sum of context force vector components to the specific direction and vorticity of a boundary object is one of the parameters to determine a dynamic process of cocreation.

The point to be emphasized in IVM is that what comes first is not a common goal or intersubjectivity among individuals, although they are necessary to be originally within a certain boundary object to start co-creation. A common goal and intersubjectivity can be developed along with co-creation. Star also criticized the ignorance of this dynamic in related researches and denies this major assumption inherent in collaboration studies as follows:

"Many models, in the late 1980s and continuing today, of cooperation (co-creation in this paper) often began conceptually, with the idea that first consensus must be reached, and the cooperation could begin. From my own field work among scientists and others cooperating across disciplinary boarders, and two historical analyses of heterogeneous groups who did cooperate and did not agree at the local level, it seemed to me that the consensus model was untrue. Consensus was rarely reached, and fragile when it was, but cooperation continued, often unproblematically. The dynamic involved in this explanation is core to the notion of boundary objects" (Star, 2010). 


\subsection{Findings}

In this study, the authors found:

- intersubjectivity among individuals is formed through the co-creation process;

- the co-creation process is directed by design contexts of each individual and contexts of each individual are also altered through the co-creation process;

- co-creativity, which authors define as a shared motivation among individuals to realize their concept, is generated through the co-creation process;

- the formation of both intersubjectivity and co-creativity are positively correlated.

\subsection{Limitation and future research}

The main limitation of this study is the simplicity of the design problem. The authors have intentionally selected a simple design problem "designing a pizza for Little Red Riding Hood to bring to her sick grandmother" because of the focus of the study as to evaluate the fundamental factors of co-creation dynamics, intersubjectivity, context and co-creativity. The second reason of selecting such design problem is, authors wanted to assign a design problem which makes the individual competency level same for every team member. When it comes to design a pizza, in general, everyone is familiar to it as a pizza delivery service system and no one knows how to do it professionally in group in fifteen minutes. Furthermore, there are other factors which the authors have recognised the importance of but hasn't taken into consideration in this study such as impact of individual and atmosphere surrounding around. Strictly speaking, this study tries to capture a basic co-creation dynamics by using a very simple situation only with few fundamental factors. As the future step of this study, more minor factors of co-creation dynamics should be considered to apply this basic model to describe more detail co-creation dynamics behaviours.

\section{Conclusion}

In this study, the authors have focused on the human factor and proposed methodologies to evaluate fundamental factors of co-creation dynamics, intersubjectivity, context, and co-creativity. To examine them, an experimental study was conducted related to the type of collaborative design process.

The authors emphasize the importance of capturing co-creation dynamics as a dynamic system containing key factors, rather than focusing on one certain key factor. Representing the knowledge obtained from the experiment, the Inverted Vortex Model is proposed to describe the dynamic mechanism of the formation process of inter-subjectivity model through co-creation.

The human factor has been usually argued from the viewpoint of collaboration; in this study, it is argued from the higher viewpoint of co-creativity. This research will contribute to the establishment of an efficient and practical methodology to form and manage sustainable collaborative projects among individuals in different contexts in various fields.

\section{Acknowledgment}

This work was supported by JSPS KAKENHI Grant Numbers JP16K16309 and JP16H03015.

\section{References}

Amabile, T.M. (1983), "The social psychology of creativity: A componential conceptualization”, Journal of Personality and Social Psychology, Vol. 45 No. 2, pp. 357-376. https://doi.org/10.1037/0022-3514.45.2.357

Bower, M. (2014), "Developing open intersubjectivity: On the interpersonal shaping of experience", Phenomenology and the Cognitive Sciences, Vol. 14 No. 3, pp. 455-474. https://doi.org/10.1007/s11097-0149346-2

Cash, P., Dekoninck, E.A. and Ahmed-Kristensen, S. (2017), "Supporting the development of shared understanding in distributed design teams", Journal of Engineering Design, Vol. 28 No. 3, pp. 147-170. https://doi.org/10.1080/09544828.2016.1274719

Chen, J.C.W. (2017), "Group creativity: mapping the creative process of a cappella choirs in Hong Kong and the United Kingdom using the musical creativities framework", Music Education Research, Vol. 20 No. 1, pp. 5970. https://doi.org/10.1080/14613808.2017.1290594 
Donohoe, J. (2016), Husserl on Ethics and Intersubjectivity: From Static and Genetic Phenomenology, University of Toronto Press, Toronto.

Gendron, E., Pourroy, F., Carron, T. and Marty, J.-C. (2012), "Towards a structured approach to the definition of indicators for collaborative activities in engineering design”, Journal of Engineering Design, Vol. 23 No. 3 , pp. 195-216. https://doi.org/10.1080/09544828.2011.576334

Gilson, L.L., Lim, H.S., Litchfield, R. and Gilson, P.W. (2015), "Creativity in Teams: A Key Building Block for Innovation and Entrepreneurship", In: Shalley, C. and Hitt, M.A. (Eds.), The Oxford Handbook of Creativity, Innovation, and Entrepreneurship, Oxford University Press, Oxford. https://doi.org/10.1093/oxfordhb/9780199927678.013.0015

Kleinsmann, M., Deken, F., Dong, A. and Lauche, K. (2012), "Development of design collaboration skills", Journal of Engineering Design, Vol. 23 No. 7, pp. 485-506. https://doi.org/10.1080/09544828.2011.619499

Maier, A.M., Kreimeyer, M., Lindemann, U. and Clarkson, P.J. (2009), "Reflecting communication: a key factor for successful collaboration between embodiment design and simulation”, Journal of Engineering Design, Vol. 20 No. 3, pp. 265-287. https://doi.org/10.1080/09544820701864402

Matsumae, A. (2014), "Multiagency University-Industry Collaboration to Innovate Traditional Japanese Architectural Industry", Proceedings of 2014 University-Industry Interaction Conference, Barcelona, Spain, April 23-25, 2014, UIIN, pp. 215-228.

Matsumae, A. and Burrow, K. (2016), "Business Model Generation Canvas as a Method to Develop CustomerOriented Service Innovation”, In: Maeno, T., Sawatani, Y. and Hara, T. (Eds.), Serviceology for Designing the Future, Springer, Tokyo, pp. 551-565. https://doi.org/10.1007/978-4-431-55861-3_38

Nagai, Y., Taura, T., Sano, K. and Yasui, A. (2010), "Internal Observation of the Design Process by Extending Reflections and Poietiques - A Methodology to Study Self-reinvestigation based on Self-formation Concluded in the Self Re-investigation Stage”, Cognitive Studies, Vol. 17 No. 3, pp. 506-524. https://doi.org/10.11225/jcss.17.506

Nonaka, I. and Konno, N. (2012), The Grammar of Knowledge Creating Management for Prudent Capitalism, Toyo Keizai, Tokyo.

Nonaka, I. and Takeuchi, H. (1995), The Knowledge-Creating Company: How Japanese Companies Create the Dynamics of Innovation, Oxford University Press.

Reid, S.E. and De Brentani, U. (2004), "The Fuzzy Front End of New Product Development for Discontinuous Innovations: A Theoretical Model”, The Journal of Product Innovation Management, Vol. 21 No. 3, pp. 170 184. https://doi.org/10.1111/j.0737-6782.2004.00068.x

Runco, M.A. and Jaeger, G.J. (2012), “The Standard Definition of Creativity”, Creativity Research Journal, Vol. 24 No. 1, pp. 92-96. https://doi.org/10.1080/10400419.2012.650092

Sawyer, R.K. (2011), Explaining Creativity: The Science of Human Innovation, 2nd ed., Oxford University Press, New York.

Shalley, C.E., Zhou, J. and Oldham, G.R. (2004), "The effects of personal and contextual characteristics on creativity: Where should we go from here?”, Journal of Management, Vol. 30 No. 6, pp. 933-958. https://doi.org/10.1016/j.jm.2004.06.007

St. John, P.A. (2007), "Interactive and Emergent Processes: Possibilities and Problems in Group Creativity", Mind, Culture, and Activity, Vol. 14 No. 4, pp. 290-293. https://doi.org/10.1080/10749030701623847

Star, S.L. (2010), "This is Not a Boundary Object: Reflections on the Origin of a Concept", Science, Technology \& Human Values, Vol. 35 No. 5, pp. 601-617. https://doi.org/10.1177/0162243910377624

Star, S.L. and Griesemer, J.R. (1989), "Institutional Ecology, Translations and Boundary Objects: Amateurs and Professionals in Berkeley's Museum of Vertebrate Zoology, 1907-39”, Social Studies of Science, Vol. 19 No. 3, pp. 387-420.

Tomasello, M. (2008), "Cultural Transmission: A View from Chimpanzees and Human Infants”, In: Schönpflug, U. (Ed.), Cultural Transmission: Psychological, Developmental, Social, and Methodological Aspects, Cambridge University Press, New York, pp. 33-47.

Woodman, R.W., Sawyer, J.E. and Griffin, R.W. (1993), "Toward a Theory of Organizational Creativity", Academy of Management Review, Vol. 18 No. 2. https://doi.org/10.5465/AMR.1993.3997517

Yang, X., Dong, A. and Helander, M. (2012), "The analysis of knowledge integration in collaborative engineering teams", Journal of Engineering Design, Vol. 23 No. 2, pp. 119-133. https://doi.org/10.1080/09544828.2011.567979

Zahavi, D. (2001), Husserl and Transcendental Intersubjectivity, Ohio University Press, Athens, Ohio.

Prof. Akane Matsumae, Associate Professor

Kyushu University, Faculty of Design

3-10-313 Yaemizo, 8490935 Saga, Japan

Email: matsumae@design.kyushu-u.ac.jp 